\title{
Readmission Rate and Causes at 90-Day after Radical Cystectomy in Patients on Early Recovery after Surgery Protocol
}

\author{
Emanuela Altobelli ${ }^{\mathrm{a}}$, Maurizio Buscarini ${ }^{\mathrm{a}}$, Harcharan S. Gill ${ }^{\mathrm{b}}$ and Eila C. Skinner ${ }^{\mathrm{b}, *}$ \\ ${ }^{a}$ Department of Urology, Campus Biomedico University of Rome, Rome, Italy \\ ${ }^{\mathrm{b}}$ Department of Urology, Stanford University School of Medicine, Stanford, CA, USA
}

\begin{abstract}
.
Background: Radical cystectomy (RC) is associated with high risk of early and late perioperative complications, and readmissions. The Enhanced Recovery After Surgery (ERAS) protocol has been applied to RC showing decreased hospital stay without increased morbidity.

Objective: To evaluate the specific causes of hospital readmissions in RC patients treated before and after adoption of an ERAS protocol at our institution.

Methods: We retrospectively evaluated the outcome of 207 RC patients on ERAS protocol at the Stanford University Hospital from January 2012 to December 2014. We focused on early (30-day) and late (90-day) postoperative readmission rate and causes. Results were compared with a pre-ERAS consecutive series of 177 RC patients from January 2009 to December 2011.

Results: In the post-ERAS time period a total of 56 patients were readmitted, 41 within the first 30 days after surgery (20\%) and 15 within the following 60 days (7\%). Fever, often associated with dehydration, was the most common reason for presentation to the hospital, accounting for $57 \%$ of all readmissions. At 90 days infection accounted for $53 \%$ of readmissions. Of all the patients readmitted during the first 90 days after surgery, 32 had positive urine cultures, mostly caused by Enterococcus faecalis isolated in $18(56 \%)$. Readmission rates did not increase since the introduction of the ERAS protocol, with an incidence of $27 \%$ in the post-ERAS group versus $30 \%$ in the pre-ERAS group.

Conclusions: Despite accurate adherence to most recent perioperative antibiotic guidelines, the incidence of readmissions after RC due to infection still remains significant.
\end{abstract}

Keywords: Cystectomy, patient readmission, infection, alvimopan

\section{INTRODUCTION}

Radical cystectomy (RC) with pelvic lymph node dissection and urinary diversion is the standard treatment for muscle invasive and high-risk non-muscle invasive bladder cancer $[1,2]$. The procedure is

\footnotetext{
${ }^{*}$ Correspondence to: Eila C. Skinner, MD, Chair, Department of Urology, Stanford University School of Medicine, 300 Pasteur Dr, Grant S-287, Stanford, CA 94305-5118, USA. Tel.: +1 650724 3332; Fax: +1 650498 5346; E-mail: skinnere@stanford.edu.
}

associated with a high risk of early and late perioperative complications, and readmissions [3].

Patient outcome improved with advances in surgical and anesthetic management, but the 90-day complications rate still exceeds 60\% [4]. The Enhanced Recovery After Surgery (ERAS) protocol has been recently applied to RC and has been shown to decrease hospital stay [5]. First introduced in colorectal surgery, the ERAS protocol aims to accelerate patient recovery through evidence-based multimodal 
pathways of perioperative care, including avoiding preoperative bowel preparation and nasogastric tube, reduced narcotic pain medication, careful fluid management, and early oral feeding and mobilization [6,7] Recent studies reporting on the 30-day outcome of RC patients on ERAS protocol have reported shorter hospital stay and fewer gastrointestinal problems with no increase in other complications or hospital readmissions compared to patients on standard clinical care pathways $[8,9]$.

In this study we wished to evaluate the specific causes of hospital readmissions in patients treated before and after adoption of an ERAS protocol at our institution.

\section{MATERIALS AND METHODS}

We retrospectively evaluated the outcome of 207 RC patients on ERAS protocol at the Stanford University Hospital from January 2012 to December 2014. Complications were reported according to the Clavien-Dindo classification system. We focused on early (30-day) and late (90-day) postoperative readmission rate and causes. Results were compared with a pre-ERAS consecutive series of 177 RC patients from January 2009 to December 2011. All postoperative complications and readmission data were collected from both local and outside hospital and physician office records.

Stanford ERAS protocol is a three-phase multimodal care pathway that includes pre-, intra- and post-operative steps, supported by published data [10] The protocol includes preoperative education, no bowel prep, 24 hour perioperative antibiotics with cefoxitin begun within 1 hour of incision, epidural postop analgesia with minimization of narcotics, careful fluid management, no nasogastric tube and early feeding. Alvimopan, a gastrointestinal opioid receptor antagonist, was introduced in the protocol in July 2013 and was used in the last 101 patients. Patients did not routinely go to the ICU and home intravenous hydration was not used. We excluded from the analysis 4 patients who remained intubated post-operatively and could not be started on oral feeding. Results were compared with a preERAS consecutive series of $177 \mathrm{RC}$ patients from the three years prior to institution of the ERAS protocol, January 2009 to December 2011. Data were analyzed with SPSS ${ }^{\circledR}$, version 19.0. A logistic regression model was performed to identify independent variables associated with readmission. The Institutional
Review Board waived requirement of a separate informed consent for this study.

\section{RESULTS}

A total of 207 RC patients between January 2012 and December 2014 were included in the post-ERAS group, and 177 in the pre-ERAS group. Details of the patient composition of each group are described in Table 1. Fewer patients in the ERAS time period underwent ileal conduit diversion ( $75 \%$ vs $63 \%)$ and robotic-assisted surgery ( $25 \%$ vs $15 \%$ ), and more received neoadjuvant chemotherapy (6\% vs $63 \%$ ). Median hospital length of stay decreased from 7 to 6 days following the institution of the ERAS program, and to 5 days with the addition of alvimopan in the last 101 patients $(p=0.001)$. Within the first 90 days postoperatively, all patients experienced at least one complication, though most were low-grade. The highest grade complication was $\geq$ Clavien grade 3 in $25 \%$ in the pre-ERAS group, and $23 \%$ in the post-ERAS group. The 90-day readmission rate was not significantly different between the pre-ERAS and post-ERAS time periods, occurring in $30 \%$ and $27 \%$ respectively.

In the post-ERAS time period a total of 56 patients were readmitted, 41 within the first 30 days after surgery (20\%) and 15 within the following 60 days (7\%). Eleven patients had multiple readmissions in the first 90 days. Median time from discharge to readmission was 15 days (range 1 to 87). Median readmission hospital stay was 4 days (range 2 to 18 ). Fever was the most common reason for readmission, accounting for $57 \%$ of all readmissions. Other causes were abdominal pain and/or gastrointestinal symptoms $(25 \%)$, dyspnea or chest pain, flank pain, or urinary retention (18\% combined).

A summary of the diagnoses for the patients with 30 and 90-day readmissions is shown in Table 2. Of the 41 patients readmitted within 30 days of surgery, infection accounted for $63 \%$ of the admissions, often associated with dehydration. Other common diagnoses were dehydration, acute kidney injury, small bowel obstruction and constipation. Of the 26 patients with at least one infection, 8 patients had febrile UTI and 11 had urosepsis. An additional $10 \%$ had a UTI diagnosed without fever during the readmission. Beside urinary infection, five patients had $C$. difficile colitis, three pelvic abscesses, and two other infections (pneumonia, cholecystitis). 
Table 1

Demographics, surgery and outcome in radical cystectomy patients, before and after the adoption of ERAS protocol without (group 1) and with Alvimopan (group 2)

\begin{tabular}{|c|c|c|c|c|}
\hline \multirow[b]{2}{*}{$\begin{array}{l}\text { Patients } \\
\text { demographics }\end{array}$} & \multirow{2}{*}{$\begin{array}{c}\text { Pre-ERAS } \\
\text { Jan } 09 \text { - Dec } 11\end{array}$} & \multicolumn{3}{|c|}{ Post-ERAS } \\
\hline & & Jan 12 - Jun 13 & $\begin{array}{l}\text { W/Alvimopan } \\
\text { Jul } 13 \text { - Dec } 14\end{array}$ & $\begin{array}{c}\text { Total } \\
\text { Jan } 12-\text { Dec } 14\end{array}$ \\
\hline Patients number & 177 & 106 & 101 & 207 \\
\hline Median age & $70(33-91)$ & $72(33-90)$ & $67(43-89)$ & $69(43-89)$ \\
\hline Male & $130(73 \%)$ & $83(78 \%)$ & $77(76 \%)$ & $160(77 \%)$ \\
\hline Female & $47(27 \%)$ & $23(22 \%)$ & $24(24 \%)$ & $47(23 \%)$ \\
\hline Median BMI (range) & $27(14-55)$ & $27(19-55)$ & $26(18-47)$ & $27(19-55)$ \\
\hline Smoking history & $118(67 \%)$ & $74(70 \%)$ & $70(70 \%)$ & $144(70 \%)$ \\
\hline \multicolumn{5}{|l|}{ Charlson comorbidity index: } \\
\hline$\leq 2$ & $84(47 \%)$ & $47(44 \%)$ & $47(46 \%)$ & $94(45 \%)$ \\
\hline$\geq 3$ & $93(53 \%)$ & $59(56 \%)$ & $54(54 \%)$ & $113(54 \%)$ \\
\hline Heart disease & $23(13 \%)$ & $21(19 \%)$ & $13(13 \%)$ & $34(16 \%)$ \\
\hline Diabetes & $58(15 \%)$ & $13(12 \%)$ & $14(14 \%)$ & $27(13 \%)$ \\
\hline Cronic kidney insufficiency & $30(17 \%)$ & $37(35 \%)$ & $22(22 \%)$ & $59(28 \%)$ \\
\hline Neoadiuvant chemo & $11(6 \%)$ & $76(72 \%)$ & $54(54 \%)$ & $130(63 \%)$ \\
\hline Prior pelvic radiation & $11(6 \%)$ & $10(9 \%)$ & $4(4 \%)$ & $14(7 \%)$ \\
\hline $\begin{array}{l}\text { Prior abdominal surgery } \\
\text { Surgery }\end{array}$ & $62(35 \%)$ & $43(41 \%)$ & $37(37 \%)$ & $80(39 \%)$ \\
\hline Robot assisted & $45(25 \%)$ & $30(28 \%)$ & $1(1 \%)$ & $31(15 \%)$ \\
\hline \multicolumn{5}{|l|}{ Diversion type: } \\
\hline Ileal conduit & $132(75 \%)$ & $79(75 \%)$ & $51(51 \%)$ & $130(63 \%)$ \\
\hline Orthotopic neobladder & $39(22 \%)$ & $25(24 \%)$ & $46(46 \%)$ & $71(34 \%)$ \\
\hline Continent cutaneous diversion & $6(3 \%)$ & $2(1 \%)$ & $4(3 \%)$ & $6(3 \%)$ \\
\hline Trasfusion $(\%)$ & $84(47 \%)$ & $38(36 \%)$ & $51(51 \%)$ & $88(43 \%)$ \\
\hline \multicolumn{5}{|l|}{ Tumor stage: } \\
\hline $\mathrm{T} 2$ or less & $232(60 \%)$ & $64(60 \%)$ & $77(77 \%)$ & $141(68 \%)$ \\
\hline $\mathrm{T} 3, \mathrm{~T} 4$ & $75(42 \%)$ & $42(40 \%)$ & $24(23 \%)$ & $66(32 \%)$ \\
\hline Nodal metastasis & $29(16 \%)$ & $24(23 \%)$ & $15(15 \%)$ & $39(19 \%)$ \\
\hline Outcome & & & & \\
\hline \multicolumn{5}{|l|}{ Median days (range): } \\
\hline Ambulation & $3(1-20)$ & $2(1-10)$ & $2(1-6)$ & $2(1-6)$ \\
\hline Flatus & $3(1-13)$ & $3(1-8)$ & $2(1-7)$ & \\
\hline Bowel movement & $5(2-19)$ & $5(3-16)$ & $4(2-7)$ & $3(2-16)$ \\
\hline Regular diet & $5(2-36)$ & $5(3-17)$ & $4(2-7)$ & $5(2-17)$ \\
\hline Hospital stay (range) & $7(3-78)$ & $6(3-27)$ & $5(3-36)$ & $6(3-36)$ \\
\hline 30-day readmission & $48(27 \%)$ & $23(22 \%)$ & $18(18 \%)$ & $41(20 \%)$ \\
\hline 90-day readmission & $53(30 \%)$ & $31(29 \%)$ & $25(25 \%)$ & $56(27 \%)$ \\
\hline 90-day mortality & $4(1 \%)$ & $2(2 \%)$ & $1(1 \%)$ & $3(1 \%)$ \\
\hline 90-day minor complications (Clavien 1-2) & $280(73 \%)$ & $79(75 \%)$ & $80(80 \%)$ & $159(77 \%)$ \\
\hline 90-day major complications (Clavien 3-5) & $104(27 \%)$ & $27(25 \%)$ & $21(20 \%)$ & $48(23 \%)$ \\
\hline
\end{tabular}

At 90 days infection accounted for $53 \%$ of readmissions. Genitourinary complications increased compared to the 30-day readmission group, from $29 \%$ to $47 \%$ of the readmissions. Urine leak and hydronephrosis were the most common GU complications in the late readmission group.

Of all the patients readmitted during the first 90 days after surgery, 32 had positive urine cultures, mostly caused by Enterococcus faecalis isolated in 18 (56\%). Other common pathogens were E. coli (28\%), Klebsiella (25\%), Staphylococcus (16\%), Candida (16\%) and Pseudomonas (9\%). Several patients had polymicrobial infections.

When we compared the incidence of the different infections between the pre-ERAS and post-ERAS group during readmissions, we found the same distribution in both cohorts, except for the UTI rate that was higher post-ERAS $(p=0.037)$. Readmission rates did not increase since the introduction of the ERAS protocol, with an incidence of $27 \%$ in the post-ERAS group versus $30 \%$ in the pre-ERAS group.

On a multivariable analysis, only age and Charlson comorbidity index correlated with the risk of readmission with a $p$ value of 0.018 (OR 0.37) and 0.042 (OR 3.33) respectively (Table 3).

\section{DISCUSSION}

The incidence of readmissions after RC is still high after the adoption of the ERAS protocol, 27\% in our 
Table 2

Causes of readmission among 56 patients in the post-ERAS group readmitted during the first 90 days from surgery. Forty-one patients readmitted between day 1 and day 30 and 15 between day 31 and day 90

\begin{tabular}{lcc}
\hline & $<30$ day $-\mathrm{n}(\%$ of 41$)$ & $31-90$ day $\mathrm{n}(\%$ of 15$)$ \\
\hline Infectious Causes & 26 patients $(63 \%)$ & 8 patients $(53 \%)$ \\
Febrile UTI (without sepsis) & $8(20 \%)$ & $2(13 \%)$ \\
Urosepsis & $11(27 \%)$ & $3(20 \%)$ \\
Abscess & $3(7 \%)$ & $1(7 \%)$ \\
C. difficile colitis & $6(15 \%)$ & $2(13 \%)$ \\
Other (pneumonia, etc) & $2(5 \%)$ & $1(7 \%)$ \\
UTI without fever & $4(10 \%)$ & $4(27 \%)$ \\
Multiple infections & 4 patients $(10 \%)$ & 3 patients $(30 \%)$ \\
Noninfectious causes & 23 patients $(56 \%)$ & 10 patients $(67 \%)$ \\
Dehydration/AKI & $15(37 \%)$ & $3(20 \%)$ \\
GU complications (urine leak, etc) & $12(29 \%)$ & $7(47 \%)$ \\
Bowel problems (SBO, constipation) & $8(20 \%)$ & $3(20 \%)$ \\
CV problems (angina, hypotension) & $6(15 \%)$ & $1(7 \%)$ \\
Tromboembolism & $2(5 \%)$ & - \\
Multiple diagnosis & 12 patients $(29 \%)$ & 5 patients $(33 \%)$ \\
\hline
\end{tabular}

Table 3

Output from a logistic regression model with readmission as the dependent variable. Age and Charlson comorbidity index score correlated with readmission

\begin{tabular}{lccccc}
\hline Readmission & Odds Ratio & Standard Error & \multicolumn{2}{c}{$P$ value } & \multicolumn{2}{c}{ 95\% Confidence Interval } \\
\hline Age & 0.372 & 0.154 & 0.018 & 0.165 & 0.841 \\
Sex & 0.777 & 0.367 & 0.595 & 0.307 & 1.965 \\
Smoking Hx & 1.116 & 0.438 & 0.779 & 0.516 & 2.412 \\
BMI & 1.101 & 0.450 & 0.813 & 0.494 & 2.455 \\
Heart disease & 1.824 & 0.976 & 0.261 & 0.639 & 5.209 \\
CKI & 0.342 & 0.218 & 0.093 & 0.098 & 1.196 \\
Diabetes & 1.759 & 1.084 & 0.359 & 0.525 & 5.889 \\
Charlson score & 3.331 & 1.966 & 0.042 & 1.047 & 10.596 \\
Prior radio-tp & 0.919 & 0.706 & 0.920 & 0.180 & 4.680 \\
Prior chemo-tp & 1.769 & 0.706 & 0.153 & 0.808 & 3.869 \\
Alvimopan & 0.507 & 0.228 & 0.133 & 0.209 & 1.228 \\
Robot-assisted & 1.286 & 0.710 & 0.648 & 0.435 & 3.798 \\
Diversion type & 1.353 & 0.585 & 0.484 & 0.579 & 3.159 \\
Pathology & 0.829 & 0.319 & 0.628 & 0.390 & 1.764 \\
Post-op abx & 0.733 & 0.363 & 0.531 & 0.278 & 1.935 \\
Tansfusion & 0.937 & 0.382 & 0.874 & 0.421 & 2.086 \\
LOS & 0.762 & 0.317 & 0.515 & 0.337 & 1.724 \\
Clavien at 90d & 4.548 & 1.863 & 0.000 & 2.037 & 10.152 \\
\hline
\end{tabular}

series. Since the introduction in colorectal surgery, ERAS protocols have been shown to improve recovery of bowel function and shorten hospital stay [5, 11-13] Advantages of the use of these pathways in $\mathrm{RC}$ patients has now been reported in a number of centers, with slight variations in the protocols used, but none has shown a decrease in readmission rates with these interventions $[5,8,9,14]$.

At our institution, we introduced an ERAS protocol in January 2012, and since the addition of alvimopan in July 2013 we have observed a decrease in median LOS of 2 days from 7 to 5 days. The potential of increased readmissions associated with earlier discharge have not been realized in other series [5] Our data similarly show that the early discharge did not result in increased readmissions, which were $27 \%$ in the pre-ERAS group versus $20 \%$ in the ERAS group at 30 days. We extended the follow-up to 90day and observed similar results in late readmissions (30\% versus $27 \%$ respectively). A recent report of 247 patients who underwent robot-assisted cystectomy showed a median initial length of stay of 9 days with a $40 \%$ 90-day readmission rate. In that series UTI was the most common cause of readmission in the continent diversion patients, and second to dehydration in the ileal conduit patients [15].

We focused on the specific causes of early and late readmissions in the hope to identify an intervention plan to reduce those readmissions. The principal cause of readmission was fever, reported in almost 
$60 \%$ of the readmitted patients, mostly caused by urinary infections. The most common pathogen was Enterococcus faecalis, isolated in almost $60 \%$ of the positive urine cultures. An E. coli infection was diagnosed in less than $30 \%$ of patients. We found a lower rate of $E$. faecalis resistance to ciprofloxacin than reported in the literature, only $11 \%$ in our patients. However, the E. coli resistance to ciprofloxacin exceeded $20 \%$. It is not clear if a change in perioperative antibiotic choice or duration might impact these acquired postoperative infections. In a recent series, Pariser et al. suggest broadening perioperative antibiotics as an approach to decreased postoperative infections. Based on institutional data, they changed from cefoxitin to broader, culture-directed antimicrobial prophylaxis including routine use of antifungals [16].

However, it is unclear if patients are indeed being infected at the time of surgery (such that perioperative antibiotics would help) or if the infection is acquired later in the hospital stay or at home. In our series, all patients present with sterile urine at surgery and in accordance with the AUA best practice guidelines, we administrated a second/third generation cephalosporin for 24 hours. The AUA guidelines also suggest the alternate use of an aminoglycoside with metronidazole or clindamycin. This combination may carry problematic renal toxicity for bladder cancer patients who often have compromised renal function. In addition, as a pilot we sent urine cultures at discharge on 50 consecutive patients and found that there was little correlation between discharge culture and the bacteria causing infection on readmission. Our fear of using broader-spectrum or longer-term antibiotics is the potential development of highly resistant organisms in this group of patients.

Other observed infections were $C$. difficile colitis (14\%), pelvic abscess (7\%), pneumonia and cholecystitis (5\%). When we compared the incidence of the different infections between the ERAS and preERAS group we found the same distribution in both the early and late complications. Only the early febrile UTI rate was higher in the ERAS group, possibly related to a higher percentage of neobladders performed in this group.

The main limitation of this study is its retrospective design. We made every effort to obtain records including all cultures for patients admitted to outside hospitals, but it is possible that some admissions were missed. This was particularly true for the comparison group prior to 2012, where in a few patients we were not able to obtain complete data about causes of readmission. In addition there are differences between the groups that could have an impact on complications, such as the percentage treated with neoadjuvant chemotherapy, undergoing continent diversion or robotic surgery. However, in our multivariable analysis we did not find that any of these factors appeared to be associated with the readmission or infection rates. Future directions will focus on the design of additional strategies to prevent and manage the development of infections after RC.

In conclusion, using the ERAS protocol with alvimopan we decreased the median hospital stay by 2 days without increasing the readmission rate. Despite accurate adherence to most recent perioperative antibiotic guidelines, the incidence of readmissions after RC due to infection still remains significant. About $60 \%$ of the UTIs in our patients were caused by Enterococcus. Prospective studies are needed to test potential interventions that may decrease the rate of hospital readmission in these patients.

\section{ACKNOWLEDGMENTS}

We thank Elizabeth A. McCormick, Data Manager, and the late John Allen, Stanford University School of Medicine, for their essential data collection support for this manuscript.

\section{CONFLICT OF INTEREST}

The authors have no conflict of interest or financial support to report.

\section{REFERENCES}

[1] Stein JP, Lieskovsky G, Cote R, Groshen S, Feng AC, Boyd S, Skinner E, Bochner B, Thangathurai D, Mikhail M, Raghavan D, Skinner DG. Radical cystectomy in the treatment of invasive bladder cancer: Long-term results in 1,054 patients. J Clin Oncol 2001;19(3):666-75.

[2] Hautmann RE, Abol-Enein H, Davidsson T, Gudjonsson S, Hautmann SH, Holm HV, Lee CT, Liedberg F, Madersbacher S, Manoharan M, Mansson W, Mills RD, Penson DF, Skinner EC, Stein R, Studer UE, Thueroff JW, Turner WH, Volkmer BG, Xu A, International Consultation on Urologic Disease-European Association of Urology Consultation on Bladder C. ICUD-EAU International Consultation on Bladder Cancer 2012: Urinary diversion. Eur Urol 2013;63(1):67-80.

[3] Kim SP, Shah ND, Karnes RJ, Weight CJ, Frank I, Moriarty JP, Han LC, Borah B, Tollefson MK, Boorjian SA. The implications of hospital acquired adverse events on mortality, length of stay and costs for patients undergoing radical cystectomy for bladder cancer. J Urol 2012;187(6):2011-7.

[4] Shabsigh A, Korets R, Vora KC, Brooks CM, Cronin AM, Savage C, Raj G, Bochner BH, Dalbagni G, Herr HW, Donat 
SM. Defining early morbidity of radical cystectomy for patients with bladder cancer using a standardized reporting methodology. Eur Urol 2009;55(1):164-74.

[5] Daneshmand S, Ahmadi H, Schuckman AK, Mitra AP, Cai J, Miranda G, Djaladat H. Enhanced recovery protocol after radical cystectomy for bladder cancer. J Urol 2014;192(1):50-5.

[6] Kehlet H. Fast-track colorectal surgery. Lancet 2008; 371(9615):791-3.

[7] Kehlet H, Wilmore DW. Multimodal strategies to improve surgical outcome. Am J Surg 2002;183(6):630-41.

[8] Dutton TJ, Daugherty MO, Mason RG, McGrath JS. Implementation of the Exeter enhanced recovery programme for patients undergoing radical cystectomy. BJU Int 2014;113(5):719-25.

[9] Karl A, Buchner A, Becker A, Staehler M, Seitz M, Khoder W, Schneevoigt B, Weninger E, Rittler P, Grimm T, Gratzke $\mathrm{C}$, Stief C. A new concept for early recovery after surgery for patients undergoing radical cystectomy for bladder cancer: Results of a prospective randomized study. J Urol 2014;191(2):335-40.

[10] Cerantola Y, Valerio M, Persson B, Jichlinski P, Ljungqvist O, Hubner M, Kassouf W, Muller S, Baldini G, Carli F, Naesheimh T, Ytrebo L, Revhaug A, Lassen K, Knutsen T, Aarsether E, Wiklund P, Patel HR. Guidelines for perioperative care after radical cystectomy for bladder cancer: Enhanced Recovery After Surgery (ERAS((R))) society recommendations. Clin Nutr 2013;32(6):879-87.
[11] Coolsen MM, van Dam RM, Chigharoe A, Olde Damink $\mathrm{SW}$, Dejong $\mathrm{CH}$. Improving outcome after pancreaticoduodenectomy: Experiences with implementing an enhanced recovery after surgery (ERAS) program. Dig Surg 2014;31(3):177-84

[12] Hahl T, Peromaa-Haavisto P, Tarkiainen P, Knutar O, Victorzon M. Outcome of Laparoscopic Gastric Bypass (LRYGB) with a Program for Enhanced Recovery After Surgery (ERAS). Obes Surg 2016;26(3):505-11.

[13] Harrison OJ, Smart NJ, White P, Brigic A, Carlisle ER, Allison AS, Ockrim JB, Francis NK. Operative time and outcome of enhanced recovery after surgery after laparoscopic colorectal surgery. JSLS 2014;18(2):265-72.

[14] Pruthi RS, Nielsen M, Smith A, Nix J, Schultz H, Wallen EM. Fast track program in patients undergoing radical cystectomy: Results in 362 consecutive patients. J Am Coll Surg 2010;210(1):93-9.

[15] Wittig K, Ruel N, Barlog J, Crocitto L, Chan K, Lau C, Wilson T, Yuh B. Critical analysis of hospital readmission and cost burden after robot-assisted radical cystectomy. J Endourol 2016;30(1):83-91.

[16] Pariser JJ, Anderson BB, Pearce SM, Han Z, Rodriguez JA 3rd, Landon E, Pisano JC, Smith ND, Steinberg GD. The effect of broader, directed antimicrobial prophylaxis including fungal coverage on perioperative infectious complications after radical cystectomy. Urol Oncol 2016;34(3): 121.e9-14. 